AC 2011-229: LOCATION IDENTIFICATION USING PASSIVE UHF RFID SYSTEM

Patrick Anderson, CET

Ghassan T Ibrahim, Bloomsburg University

Associate professor at the Physics and Engineering Technology Department. Research interest is in the field RF communication.

(C)American Society for Engineering Education, 2011 


\title{
LOCATION IDENTIFICATION USING PASSIVE UHF RFID SYSTEM
}

\begin{abstract}
Radio Frequency Identification (RFID) has been widely utilized and applied in retail stores and manufacturing environments. Over the years the advances in the technology provided the means for a wide range of intriguing applications, one of which is the location estimation of stationary objects. In a warehouse environment the identification of the stored items' locations in predefined shelves is of an essence. With hundreds or even thousands of stored items the tracking, locating, and retrieving one single item can be time consuming and costly, especially if the items are misplaced. A cost effective system is required to tag the stored items and automatically identify their locations. The system of choice was a passive ultra high frequency (UHF) RFID system utilizing very low cost tags, relatively long detection range and high rate of detection rate makes it most suitable for real time detection and tracking. In this paper the viability of the passive UHF RFID system to locate the position of a tagged objects, stored in a multiple shelved warehouse environment is investigated. A pilot system was implemented and its performance was investigated by a team of two students in the electronics engineering technology (EET) program during one semester senior design project. A $915 \mathrm{MHz}$ RFID system performance was investigated and analyzed in a simulated environment in the radio frequency (RF) laboratory. The concept of using RFID system for position identification in the simulated storage environment was successfully proven. The pilot system was able to identify tagged items' locations with very high efficiency especially in an RF friendly environment. The work required the analysis of the RFID interrogator (reader) antenna and the passive RFID tags radiation patterns, and a methodical RF survey of the surrounding environment.

It is emphasized that the senior design project has provided an excellant environment for the students to integrate all the knowledge gained during their educational period and apply it to implement a system utilizing a state of art technology in RF wireless communication. It provided a practical hands-on learning opportunity, and developed their ability to analyze and provide solutions for real world problems. The paper presents the experimental findings, their analysis, and the project educational outcomes.
\end{abstract}

\section{Introduction}

Projects investigating state of art technology are of vital importance for the graduating engineering technologists. In addition to the theoretical knowledge provided through the classical lectures and labs approach; these projects are valuable learning tools that provide a venue for the students to utilize their knowledge effectively, acquire in-depth understanding of the concepts, think critically and independently analyze real life problems. In the senior design project course, a group of students were assigned a project to investigate the viability of utilizing passive Ultra High Frequency (UHF) Radio Frequency Identification (RFID) system for location identification of stored items within a warehouse inventory. The passive UHF RFID tags are very small, and low cost and energy efficient, and their high data transmission speed makes them suitable for real time tracking and identification ${ }^{1,2}$. The tags are passive, i.e. they are powered by the 
surrounding radio frequency $(\mathrm{RF})$ field which is radiated by the reader (interrogator) antenna located in the vicinity of the tag. Advances in the RFID technology have reached such a state where they are now widely used in various aspects of industry, giant retail stores, and large scale warehouses.

RFID systems applications in location estimation of stationary objects have been investigated by various authors. They introduced methods for the localization of passive tags within a predetermined interrogation range of the reader and presented methods to estimate the distance of the RFID tag from the reader ${ }^{3}$. Koutsou ${ }^{4}$ used an RFID reader with the tags distributed at a number of known positions; utilizing the received signal strength (RSS) the tags positions were estimated using a Bayesian localization method. Others used a probabilistic method to determine the position of RFID tags to localize robots and persons within their environment ${ }^{5}$. RFID systems have reliability concerns, especially when using the low power and low cost tags. These were addressed ${ }^{6}$ when a system was used to track moving parts or part carriers in an industrial environment. Multiple RFID readers with low cost tags were used in an effort to define a precise location of an object ${ }^{7,8,9}$. All these investigations were aimed to determine, with a reasonable precision, the distance of an object from the reader antenna However their suitability to be used and applied practically in a warehouse environment are limited mostly due to cost.

In a warehouse environment the identification of the stored items' locations in predefined shelves is of an essence. With hundreds or even thousands of stored items the tracking, locating and retrieving one single item can be time consuming and costly, especially if the items are misplaced. In this paper the viability of the passive $915 \mathrm{MHz}$ UHF RFID system to locate the positions of tagged items, stored in a multiple shelved warehouse environment is investigated. As a proof of concept a pilot model, consisting of two three shelf racks for storing small boxes identified using passive UHF tags, was assembled in a laboratory environment. The system performance was investigated and analyzed by a team of two students, in the four year electronics engineering technology (EET) program, during a one semester senior design project. The paper starts with an overview of the passive RFID systems, and then introduces the experimental approach and the analyses performed leading to the development of a unique location identification technique,

\section{UHF Passive RFID System Overview}

The two main system components of an RFID system are the RFID reader, and the RFID tags which are attached to the items to be identified (merchandise, people, pets, furniture, instruments etc.), see figure 1. One type, the passive UHF RFID system, is characterized by its agility, relatively long range, very high read rates, and low cost passive tags. The passive tag, which is energized by the electromagnetic RF field transmitted by the reader, is composed of an antenna, an integrated silicon chip that includes the basic modulation circuitry and a non-volatile memory. The RF signal, called the carrier signal, is transmitted by the reader at the hopping frequency band of 902-922 MHz. When the RF field reaches the tag it couples with the tag's antenna coil and consequently an AC voltage is generated across the coil. This voltage is rectified to supply power to the tag. As the tag is activated it starts to transmit back the coded information stored in its memory to the reader, using backscattered modulation technique, ${ }^{1}$ where it is decoded and retrieved. 


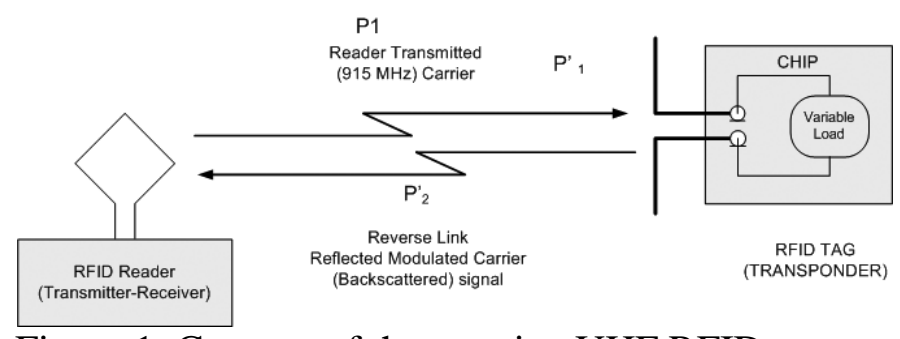

Figure 1, Concept of the passive UHF RFID system

\section{Experimental Setup}

A two column three level simple storage model was set-up and placed on a wooden lab bench one meter above the floor. Column A has three shelves labeled from top A1, A2, and A3 and column B shelves labeled B1, B2 and B3. The inventories used were empty carton boxes; each box labeled using a passive RFID tag with a unique assigned name written into the tag memory. The location identification process utilizes the ALIEN UHF Passive RFID system ${ }^{10}$ consisting of one ALIEN 9900 reader (interrogator) and four ALR-9611 antennas placed around the storage model. The reader communicates with the control and monitoring computer through the serial RS232 port. A Visual Basic (VB) program was developed; it configures the ALIEN 9900 reader built-in gateway software commands and collects, in real time, the reads/sec data from the four antennas and stores them in an array. The stored data are then processed to identify the names and locations of the tags.

The read/sec data are determined by the RFID reader's internal software. The reader, controlled by the computer through the VB program, scans sequentially through the antennas and transmits a coded RF carrier signal which activates and interrogates the passive tags within the antennas' field. Once powered, the tags transmit their information back to the reader at a very high rate; the number of times that the reader actually records the received data is cataloged as "counts". The reads/sec are calculated as the number of accumulated counts over the span of one second, which decrease considerably as the data is transferred between the reader Gateway software and the computer to be processed by VB software. The tag's RSS and the resulting $\mathrm{read} / \mathrm{sec}$ value is dependent upon multiple variables including: the radiation pattern of the reader antennas, the tag radiation pattern, the orientation of the tag in relation to the reader antenna, the reader antenna signal level, and its distance from the tag.

\section{Data Collection Process and its Reliability}

The VB program collects and analyzes the received tags data to determine the names and the actual locations of the tagged items. In essence, the analyses are based on the tags' received RSS detected by the reader antennas, which is qualitatively estimated utilizing the reads/sec data. To justify the effectiveness of the qualitative data collection and analysis technique the VB software, together with ALIEN RFID system, was tested in the lab environment free of RF reflecting equipment, to regenerate the ALN 9540 tag radiation pattern using the read/sec data. Its shape was then compared to the corresponding manufacturer radiation pattern, which is produced under constrained test conditions in anechoic chamber isolated from real world physical effects using RSS measurements. 
The profile of the ALN-9540 tag radiation pattern was determined by monitoring the average $\mathrm{read} / \mathrm{sec}$ collected by the software at various angular rotations of the tag with respect to the stationary test antenna (TA), which is a reader antenna. This was accomplished by positioning the tag on a wooden pole and rotating it horizontally by 22.5 degrees increments see figure 4 . At each angular position the recorded data was based on the average value of five sets of reads/sec. The generated radiation pattern is shown in figure 2, together with the manufacturer's data sheet radiation pattern, figure 3 .

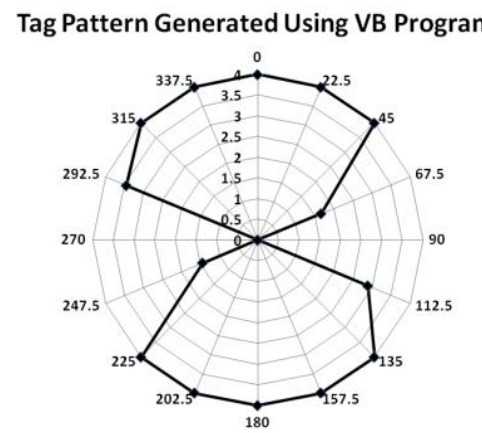

Figure 2,

Tag pattern generated using VB Reads/Sec. vs. rotational angle

ALN-9540 Inlav Sensitivitv \& Orientation World Tag Angular Sensitivity (dB vs. Angle Position)

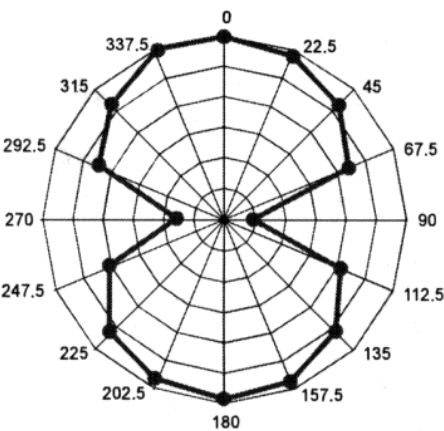

Figure 3,

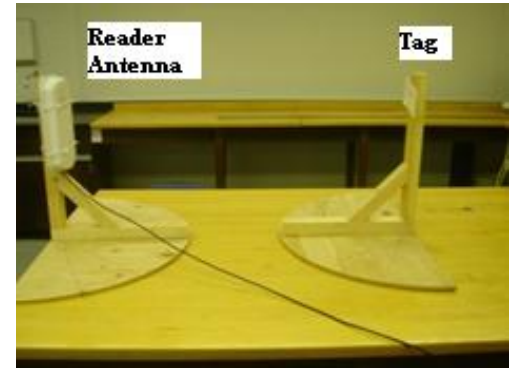

Figure 4,
Tag Manufacturer radiation pattern ${ }^{1}$
Tag radiation pattern test set up

Comparison of the two patterns clearly indicates that the proposed method, using the collected read/sec data, was successful in reproducing the tag's radiation pattern and clearly verifies that the reads/sec. data are a viable qualitative indicator of the tags received signal strength levels. Consequently the read/sec data can be effectively processed and utilized by the VB monitoring program to determine the names and locations of the individual tags.

\section{The Choice of Suitable Tag}

The ALN-9640 (a newer version of 9540) passive RFID tag was the tag of choice. This was based on analysis of the experimental results presented in figure 5. To determine the most sensitive tag (i.e. the one which requires the least amount of reader power to be activated), four tags were tested in a lab environment similar to the one in the previous test, see figure 4 . Each tested tag was placed at a distance of one meter from the reader antenna and oriented such that its maximum radiated power direction is in line with that of the reader antenna. The RF power radiated by the reader antenna was $30 \mathrm{dBm}$ (1 watt). As the power level is attenuated, it can be seen that the ALN-9640 is the most sensitive tag compared to the other tested tags since it requires the least RF signal power level to power up and transmit back its information. 


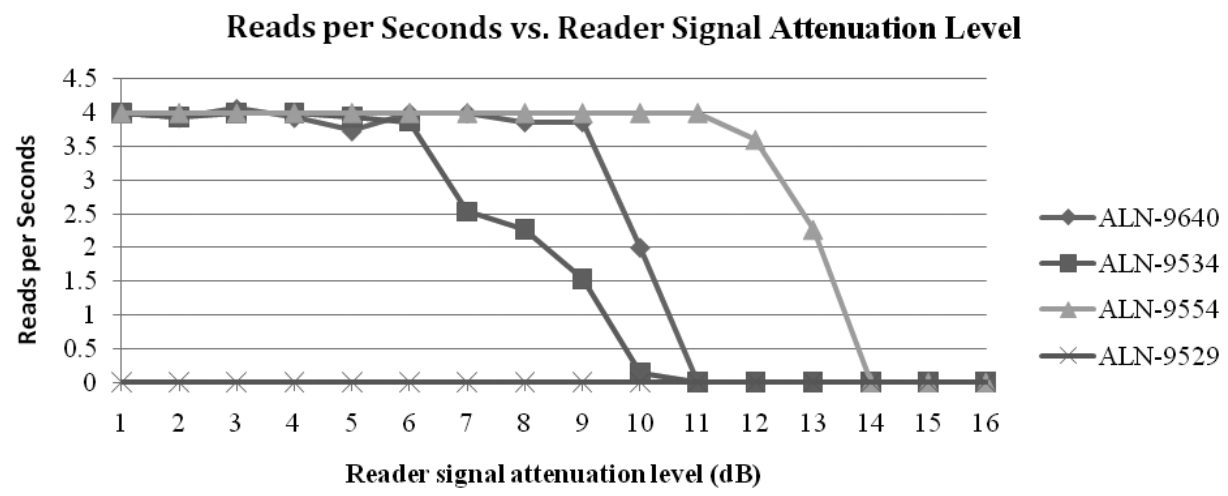

Figure 5, Tags sensitivity test

\section{$\underline{\text { Tag Performance Analysis }}$}

The performance of the ALN-9640 was analyzed to determine its sensitivity under two operating conditions. The sensitivity test was repeated with the ALN-9640 located at different distances from the reader antenna. Figure 6 shows the degradation of the sensitivity when the tag was placed further away from the reader antenna, which is a typical propagation characteristic of $\mathrm{RF}$ signal. Figure 7 shows the degradation of the tag sensitivity when it was placed in an environment cluttered with RF signal reflecting metal cabinets and equipment (RF unfriendly environment). This was attributed mainly to the multipath reflection phenomenon of the high frequency RF signal as it travels between the tag and the reader antenna. These results were important; it helped the students choose the proper operating environment for the system, and optimize the relative orientation and location of the tag with respect to the reader antennas.

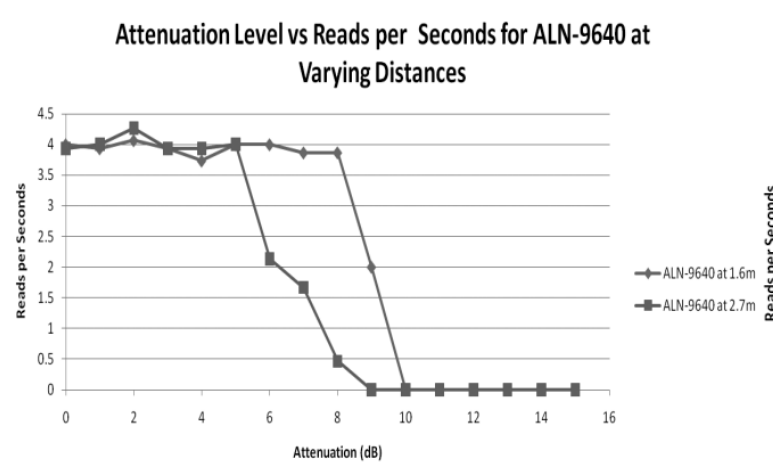

Figure 6 , Tag sensitivity at varying distances

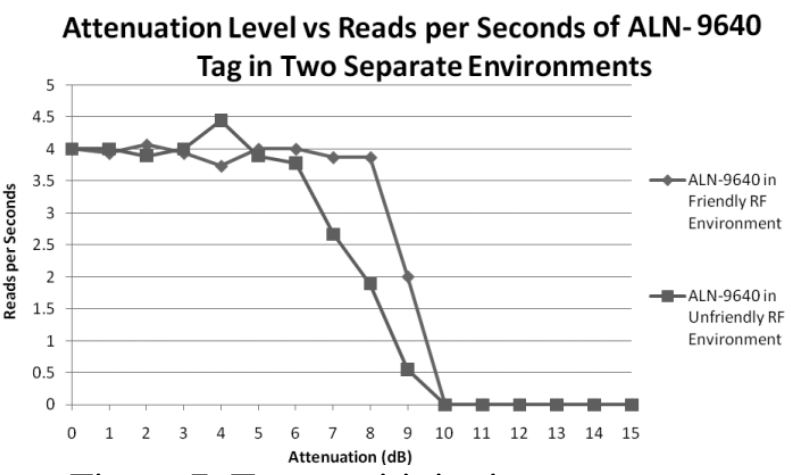

Figure 7, Tag sensitivity in two different environments

\section{Location Determination Technique}

To determine the exact locations of the tagged items on the individual shelves a methodical RF surveys and tests were performed to achieve a unique and distinct reads/sec pattern for each rack location. The surveys and tests were performed using different locations and orientations of the 4 antennas around the two 3 shelves racks, see figure 8 . The tags were placed on top of the boxes in the center of the shelves with a fixed orientation with respect to the reader antennas. 


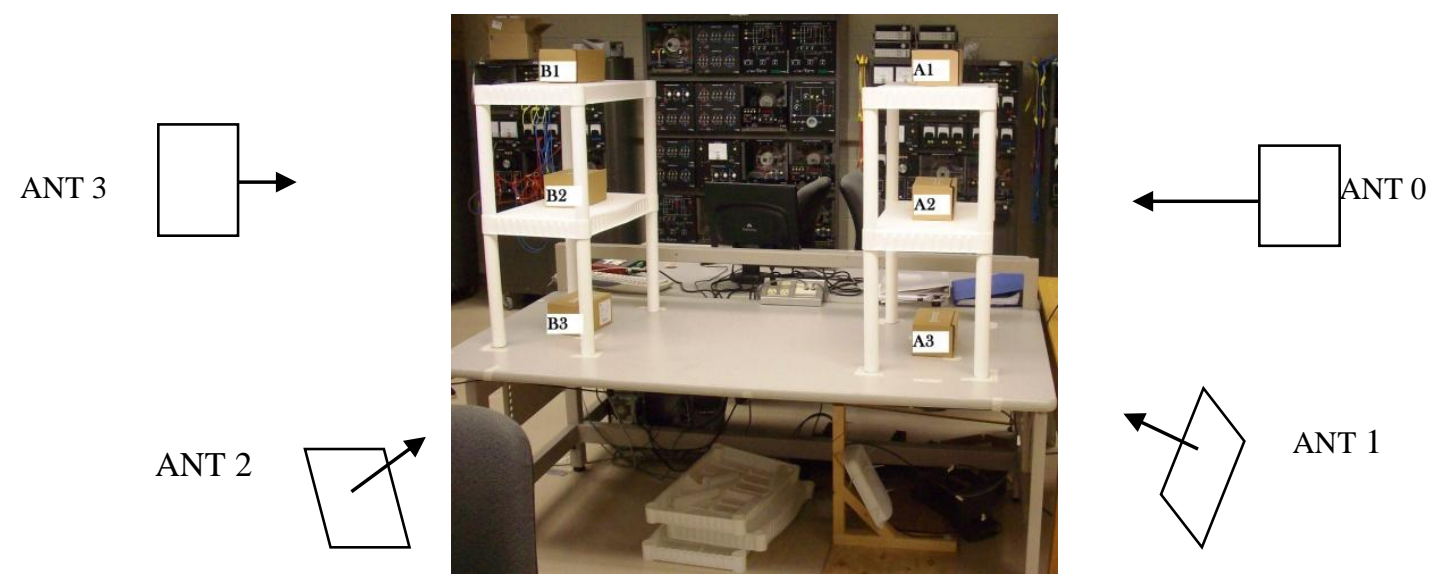

Figure 8, Antennas and Rack Setup

Each antenna was oriented such that its line of sight (LOS) with each of the individual tagged boxes ensures a distinct alignment of its radiation field patterns with the tags radiation patterns; thereby producing a distinct reads/sec for each location. The reader signal strength was set to 23 $\mathrm{dBm}$, and the racks were spaced at $80 \mathrm{~cm}$ apart. As the system was activated, each individual antenna acquired data from all the six storage locations. The acquisition process was repeated five times; and the final reads/sec data for each individual location was the average value of the five readings.

The surveys and tests were performed at two different lab environments. The first environment was cluttered with RF reflecting equipment and panels (RF unfriendly environment). With one tagged box placed on each of the six shelves, they were scanned sequentially by the four antennas " 0 to 3 ". The averaged reads/sec collected by each antenna, for the six A and B locations, were recorded and stored in an array, see table 1.

Table 1, Read/sec data recorded for each box in an RF unfriendly environment

\begin{tabular}{|c|c|c|c|c|}
\hline \multirow{2}{*}{ Location } & \multicolumn{4}{|c|}{ Average Reads/sec } \\
\cline { 2 - 5 } & Antenna 0 & Antenna 1 & Antenna 2 & Antenna 3 \\
\hline A1(Box 1) & 4 & 0 & 0 & 0 \\
\hline A2(Box 2) & 3.934 & 3.066 & 0 & 066 \\
\hline A3(Box 3) & 4 & 4 & 0 & 4 \\
\hline B1(Box 4) & 0 & 0 & 0.798 & 3.6 \\
\hline B2(Box 5) & 1.332 & 0 & 4 & 2.534 \\
\hline B3(Box 6) & 0.732 & 1.998 & 2.332 & \\
\hline
\end{tabular}

From table 1 A distinct read/sec pattern was observed for each tagged box, antenna 0 acquired high reads/sec form the tagged boxes located in A1, A2, and A3, antenna 1 acquired high reads/sec from the tagged boxes in A2, A3, and B3. Antenna 2 acquired high reads/sec from locations B2 and B3 and low read/sec from B1, but never detected the tags on A1 and A2 and A3. However, the reads/sec values were continuously varying as the process repeated thereby causing uncertainty in generating a consistent repetitive stable pattern necessary to identify the locations. This was attributed mainly to the multipath reflections of the RF signals generated by the reader and tag antennas. 
To reduce the multipath effects some of the RF reflecting objects removed from the immediate vicinity of the antennas and the process was repeated; the average reads/sec acquired by each antenna for the six different locations is tabulated in table 2. The data patterns acquired by the four antennas for each location were more consistent, distinct, and repetitive.

Table 2, Average Antenna Readings with RF reflecting equipment removed

\begin{tabular}{|c|c|c|c|c|}
\hline \multirow{2}{*}{ Location } & \multicolumn{4}{|c|}{ Average Reads/sec } \\
& Antenna 0 & Antenna 1 & Antenna 2 & Antenna 3 \\
\hline A1(Box 1) & 5.598 & 0 & 0 & 0 \\
\hline A2(Box 2) & 5.866 & 4.066 & 0 & 1.998 \\
\hline A3(Box 3) & 5.332 & 5.332 & 0 & 0 \\
\hline B1(Box 4) & 0 & 0 & 0 & 3.33 \\
\hline B2(Box 5) & 3.936 & 0.066 & 5.598 & 5.868 \\
\hline B3(Box 6) & 0.332 & 5.532 & 5.598 & 5.598 \\
\hline
\end{tabular}

Having configured the antenna locations for a consistent repetitive generation of read/sec data pattern, the pattern was mapped into a digital signature, see table 3, by setting a fixed threshold e.g. $2 \mathrm{reads} / \mathrm{sec}$. If a data $>2 \mathrm{reads} / \mathrm{sec}$ the corresponding cell is set to true " 1 ", otherwise it is false " 0 ". This digital signature was then used to automatically identify the stored boxes locations.

Table 3, Digital signature pattern

\begin{tabular}{|l|l|l|l|l|}
\hline Location & $\begin{array}{l}\text { Ant } \\
0\end{array}$ & $\begin{array}{l}\text { Ant } \\
1\end{array}$ & $\begin{array}{l}\text { Ant } \\
2\end{array}$ & $\begin{array}{l}\text { Ant } \\
3\end{array}$ \\
\hline A1(Box 1) & 1 & 0 & 0 & 0 \\
\hline A2(Box 2) & 1 & 1 & 0 & 1 \\
\hline A3(Box 3) & 1 & 1 & 0 & 0 \\
\hline B1(Box 4) & 0 & 0 & 0 & 1 \\
\hline B2(Box 5) & 1 & 0 & 1 & 1 \\
\hline B3(Box 6) & 0 & 1 & 1 & 1 \\
\hline
\end{tabular}

However, using this simple fixed threshold decision criterion could not generate a repeatable constant digital pattern. Based on the repetitive data analysis it was noted that the averaged tags reads/sec data were slightly fluctuating but always remain within the same proportion to each other. Consequently the decision criterion was modified using a variable threshold setting technique, see figure 9. The new decision criterion was based on determining the highest $\mathrm{read} / \mathrm{sec}$ value throughout the array and setting it as the threshold to which all other values were compared. The set percent values in proportion to the highest reads/sec, see figure 9 , were determined empirically such that to achieve a constant and repetitive digital signature pattern. This enabled the detection system to adapt to the slightest fluctuations in the average read/sec values. 


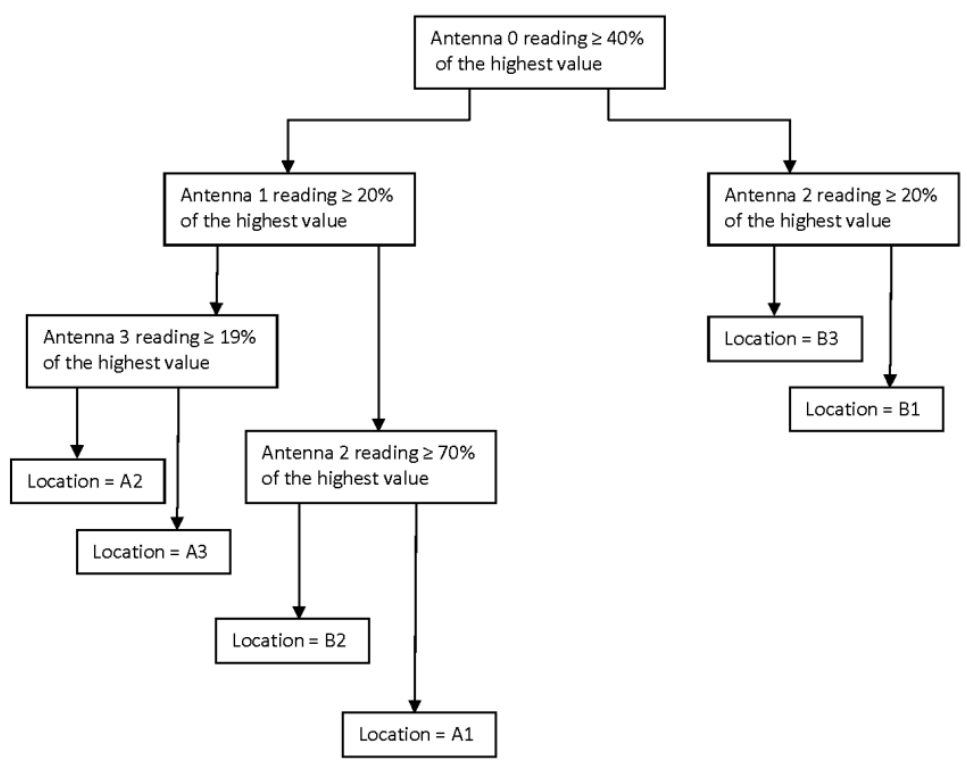

Figure 9, Tree Diagram of variable threshold criterion for Location Identification

\section{$\underline{\text { Location Identification Efficiency }}$}

The ability of the system to efficiently and automatically locate an item in a stored location was determined by measuring its reliability through a number of trials. The reliability is defined as the percentage of a number of successful trials during which the system can locate the tagged boxes in the proper rack locations. Two reliability tests were performed at two different lab environments.

The first reliability test was performed in a lab environment where a large number of RF reflecting equipment cluttered in the vicinity of the test system antennas and around the storage model. The test procedure is based on the assumption that each rack will house one tagged box, i.e. six boxes with six possible locations; consequently there will be 63 possible combinations for the tags locations. The test started with one box placed in one location at a time, then two boxes, then three, etc. For each box combination five sets of reads/sec data was recorded, each set was then processed to determine the tags locations. The outcome of a successful determination of the tag location was recorded as true, and false if unsuccessful. If a tag location was not successfully determined three times out of the five repetitive trials, a more in-depth analysis was performed in order to determine the cause. As a result the adjustment (tuning) of the detection threshold percentages in the program logic code was found to be the important factor in increasing the number of successful detection of the tag. Figure 10 shows the overall percentages for each group of data. 


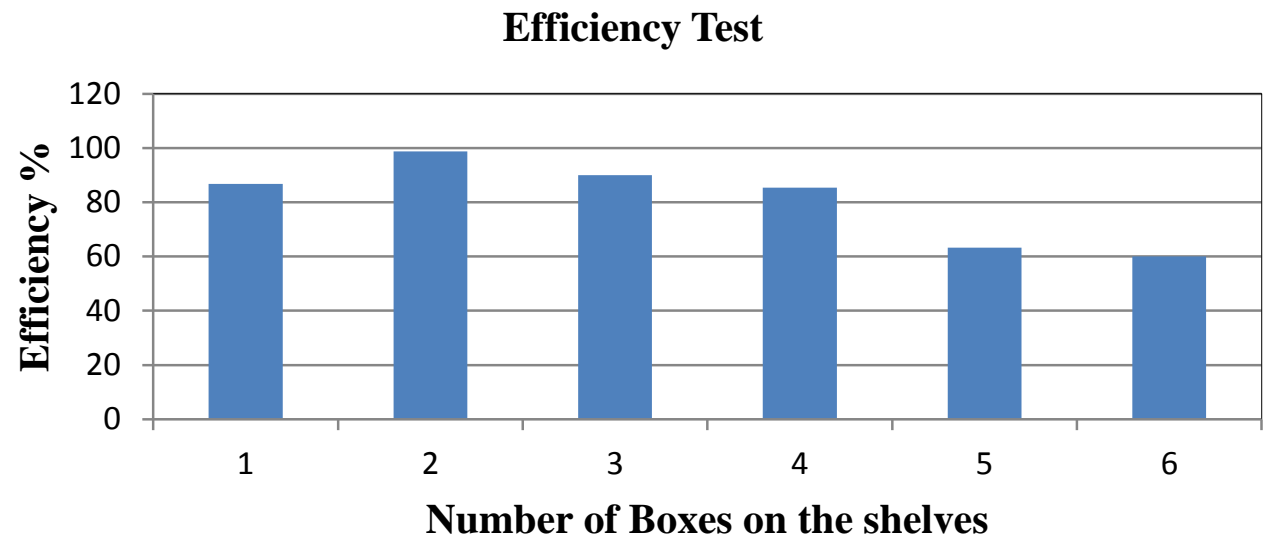

Figure 10, Overall percentages of successful trials for each location

From figure 10 it can be seen that the system efficiency decreases when there are more boxes on the shelves. Based on the previous RF survey analysis, the low efficiency of the system was attributed to the laboratory RF unfriendly environment which causes multipath reflections and dead spots. In this case the main problem was attributed to data collected through antenna 3, which was located on top of a metal box. Removal of the metal box improved the detection efficiency, see figure 11 .

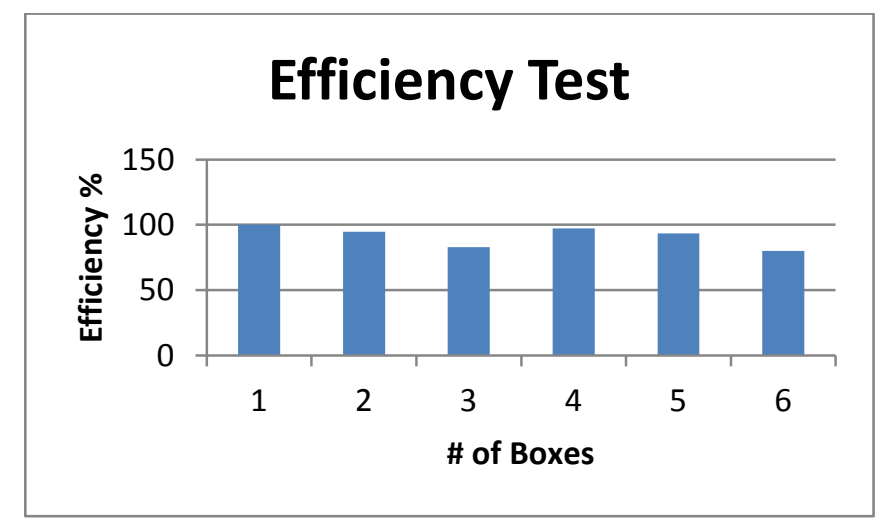

Figure 11, The improvement in efficiency due to removal of RF reflecting metal box

Based on the above findings a second more spacious environment, figure 12, was selected allowing the rack antenna configuration to be located at a far enough distance from equipments, computers, and metal cabinets thereby reducing the affect of RF multipath reflections. 

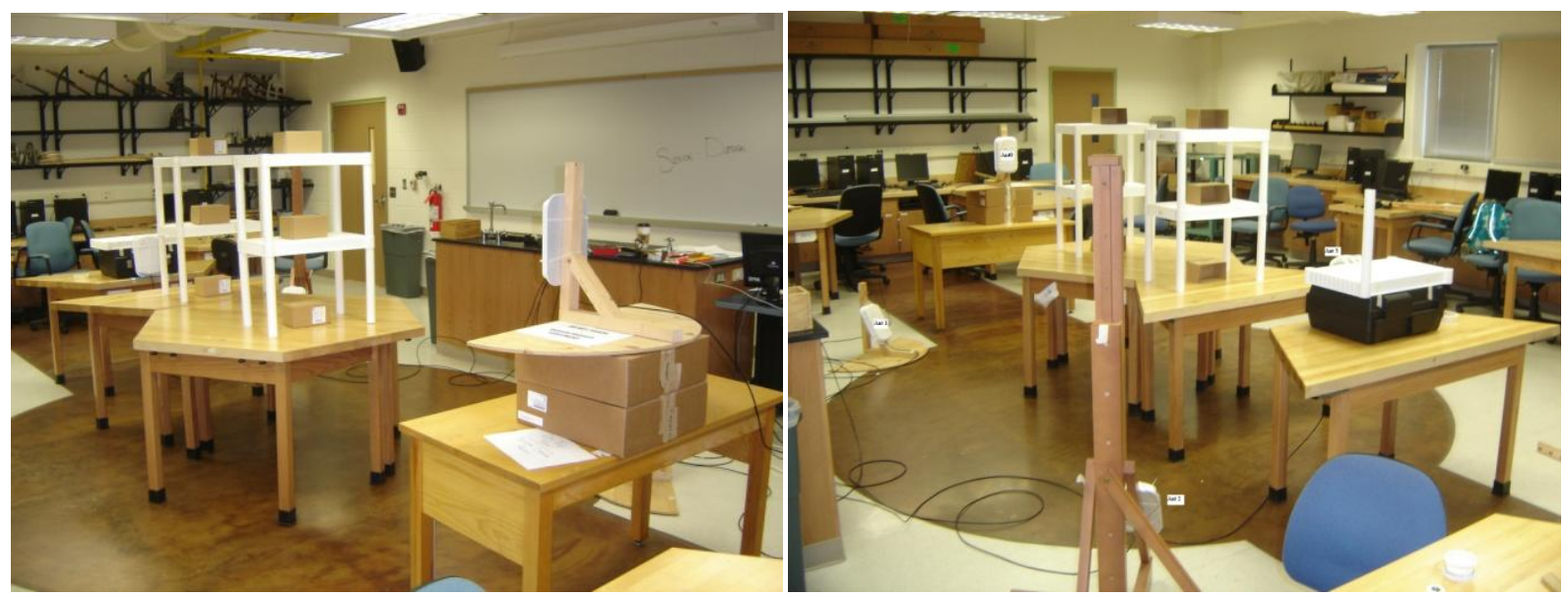

Figure 12, Second lab environment

After repositioning the racks and the antennas as in the first lab environment a similar RF survey was repeated to obtain a distinct $\mathrm{read} / \mathrm{sec}$ pattern and the threshold percentages were tuned to generate the digital signature pattern. With all the six tagged boxes placed in separate shelves and using the VB program, the averaged reads/sec data are shown in Table 4.

Table 4, Data from experiment in second location

\begin{tabular}{|c|c|c|c|c|}
\hline Location & $\begin{array}{c}\text { Average Readings } \\
\text { Antenna 0 }\end{array}$ & $\begin{array}{l}\text { Average Readings } \\
\text { Antenna 1 }\end{array}$ & $\begin{array}{l}\text { Average Readings } \\
\text { Antenna 2 }\end{array}$ & $\begin{array}{l}\text { Average Readings } \\
\text { Antenna 3 }\end{array}$ \\
\hline A1 & 1.066 & 2.2 & 0 & 0 \\
\hline A2 & 5.332 & 0 & 0 & 5.466 \\
\hline A3 & 4.8 & 3.802 & 0 & 5.332 \\
\hline B1 & 0 & 0 & 0 & 2.934 \\
\hline B2 & 5.332 & 0 & 2.068 & 5.332 \\
\hline B3 & 0 & 0 & 2.666 & 4.6 \\
\hline
\end{tabular}

The acquired read/sec values were stable and repetitive. Reliability tests were repeated for all possible combinations of the tags locations. The results of these tests see Figure 13 below, show $100 \%$ system efficiency in determining the location of the individual tagged boxes.

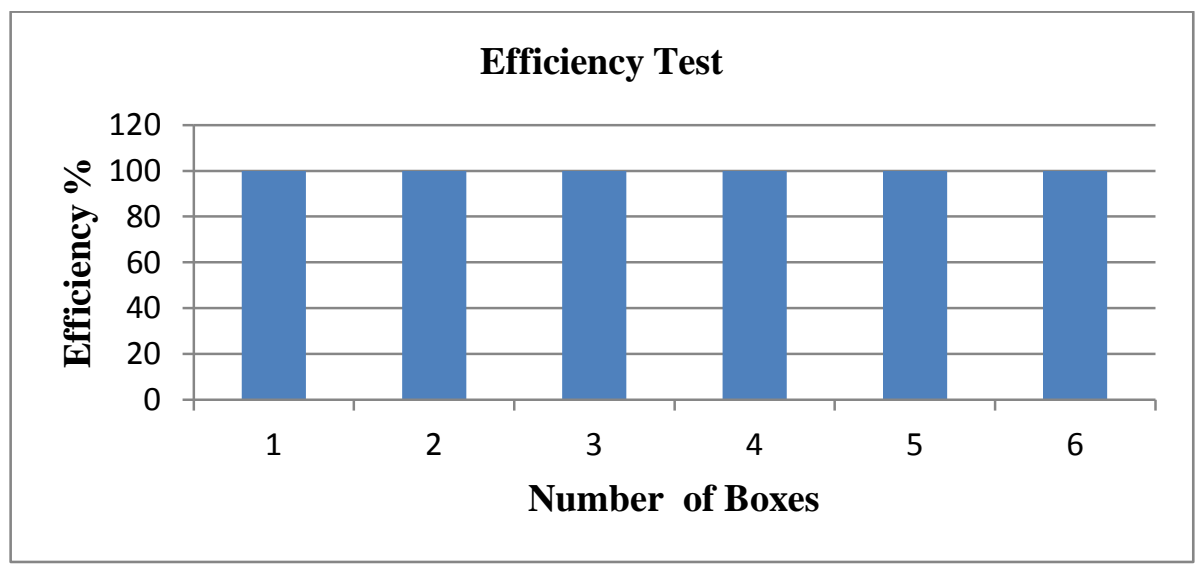

Figure 13, Efficiency test in an RF friendly environment 


\section{$\underline{\text { Summary }}$}

The concept of using RFID system for position identification in the simulated storage environment was successfully proven. The highest detection efficiency occurred in an RF friendly environment; i.e. no reflecting objects in the vicinity of the system. The following are some important guidelines to ensure a successful pilot system performance:

- The racks must be of non RF reflecting or absorbing material and at a fixed distance apart during the tests.

- The tagged boxes contain non-RF reflecting material when used with the chosen tag.

- The boxes must be placed on the shelf, see figure 14, such that to ensure a consistent orientation of the tags with respect to the surrounding antennas.

- An RF survey must be conducted; concurrently the logic thresholds within the VB program need to be adjusted to optimize the system performance.

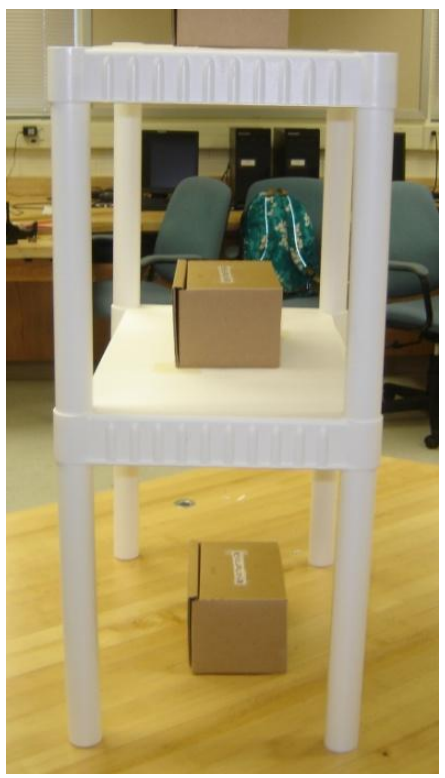

Figure 14, The Boxes with their tag orientation on the racks.

\section{Lessons Learned}

The senior design project requires the students to develop and implement a working industrial like product utilizing the knowledge gained and the hands on experience acquired during their educational years in the electronic engineering technology program. The students utilized a new evolving state of the art technology to develop and implement an automated solution for a complex industrial problem. They effectively utilized their theoretical and practical knowledge, gained during the RF Effects and Measurement course, to analyze an existing industrial problem. They were confronted with the practical complexity of RF signal propagation, and used their knowledge to provide a successful solution. They learned the theory of RFID system, gained hands-on experience on its practical applications, and how a seemingly simple operational configuration may cause considerable effects on the reliability of the system. It made the students think like engineering technologists, they did not only learn how a sophisticated system operated but they also learned how to analyze the problems associated with RF systems 
operations, signals, and their effects. Using their industrial level computer programming and mathematical background, they developed an effective VB application program, which not only acquires and analyzes the data but also controls the process and provides an excellent user interface. They also developed an effective decision based method to automatically identify the locations of the tags, and provided a reliability analysis method to justify the outcome.

\section{Conclusion}

The concept of using RFID system for position identification in a simulated storage environment was successfully proven. The tagged items' locations were successfully identified especially in an RF friendly environment. The efficiency is considerably reduced in an RF unfriendly environment due to multipath reflections and the creation of $\mathrm{RF}$ dead spots. However, further work is required to verify the applicability of the concept to monitor larger number of shelves in real world storage environment using real world items. Most important is the experience gained by the students, not only technically but they learned that a well coordinated team work is essential to the success of any project. At the end of the course some of the students' remarks are summarized below:

- Performing a methodical RF survey was essential to the successful implementation of the RFID based location identification pilot system.

- They suggested incorporating an experiment in the RF course, which they concurrently attended, to emphasize the importance of RF survey and the problems associated with RF signal propagation.

- For future work, they suggested to further evaluate the system based on a replicate of a local storage depot environment.

\section{References}

[1]. Klaus Finkenzeller, RFID Handbook second edition, WILEY, p145-148, 2003.

[2]. The RF in RFID A radio - oriented introduction to RFID, Daniel M. Dobkin and Titus $\backslash$ Wandinger, (5/17/05).

[3]. P. Wilson, D. Prashanth, and H. Aghajan, "Utilizing RFID Signaling Scheme for Localization of Stationary Objects and Speed Estimation of Mobile Objects", IEEE International Conference on RFID, March 2007

[4]. A. D. Koutsou, F. Seco, A. R. Jimenez, J. O. Roa, J. L. Ealo, C. Prieto, and J. Guevara, “ Preliminary Localization Results with an RFID based Indoor Guiding System”, IEEE International Symposium on Intelligent Signal Processing, Spain, Oct. 2007

[5]. M, Philipose, K. P. Fishkin, Intel Research Seattle, D, Fox, university of Washington, D. Hahnel, W. Burgard University of Freiberg, "Mapping and Localization with RFID Technology", Intel Research Corporation December 2003

[6]. J. Brusey, C. Floerkemeier, M. Harrisoon, and M. Fletcher, “ Reasoning about Uncertainty in Location Identification with RFID, AUTOID LABS ( http:// www.autoidcenter.org), 2006

[7]. Hinske, S., Langheinrich, M.: An RFID-based Infrastructure for Automatically Determining the Position and Orientation of Game Objects in Tabletop Games. In:Magerkurth, C., Röcker, C. (Eds.): Concepts and Technologies for Pervasive Games - A Reader for Pervasive Gaming Research. Vol. 1, Shaker Verlag, pp. 311-336 (2007) 
[8]. Zhi-yuan, Zhu; He, Ren; Jie, Tan; A method for optimizing the position of passive UHF RFID tags, 2010 IEEE International Conference on RFID-Technology and Applications (RFID-TA), Jul 2010

[9]. In:Magerkurth, C., Röcker, C. (Eds.): Concepts and Technologies for Pervasive Games - A Reader for Pervasive Gaming Research. Vol. 1, Shaker Verlag, pp. 311-336 (2007)

[10]. Alien Technology Product Overview, www.alientechnology .com 